\title{
Early closure of the protective ileostomy after rectal resection should become part of the Enhanced Recovery After Surgery (ERAS) protocol: a randomized, prospective, two-center clinical trial
}

\author{
Stanisław Kłęk ${ }^{1}$, Magdalena Pisarska ${ }^{2,3}$, Katarzyna Milian-Ciesielska ${ }^{4}$, Tomasz Cegielny ${ }^{1}$, Ryszard Choruz ${ }^{1}$, \\ Jerzy Sałówka ${ }^{1}$, Piotr Szybinski ${ }^{1}$, Michał Pędziwiatr ${ }^{2,3}$ \\ ${ }^{1}$ General and Oncology Surgery Unit, Stanley Dudrick's Memorial Hospital, Skawina, Poland \\ ${ }^{2} 2^{\text {nd }}$ Department of General Surgery, Jagiellonian University Medical College, Krakow, Poland \\ ${ }^{3}$ Department of Endoscopic, Metabolic and Soft Tissue Tumors Surgery, Krakow, Poland \\ ${ }^{4}$ Department of Pathology, Jagiellonian University Medical College, Krakow, Poland
}

Videosurgery Miniinv 2018; 13 (4): 435-441 DOI: https://doi.org/10.5114/wiitm.2018.79574

\begin{abstract}
Introduction: Protective loop ileostomy (PLI) is used to reduce the anastomotic leak rate after resection of the rectum. It is an effective, yet burdensome procedure contradicting the aims of enhanced recovery after surgery (ERAS) by slowing down recovery. Early closure (EC) of the PLI has the potential to change the situation, and it should become part of ERAS.

Aim: To analyze the effectiveness of EC in ERAS patients.

Material and methods: A randomized clinical trial was performed between October the $1^{\text {st }}, 2016$ and December the 315t, 2017. Fifty-eight adult patients (24 females, 34 males, mean age: 55.7 and 56.2) operated on for rectal carcinoma according to the ERAS protocol with PLI were randomly assigned to the late (L) or early (E) closure group (14 days after discharge). Time to start adjuvant chemotherapy, complication rate, and health care costs were analyzed. Results: There were no significant differences between groups regarding the length of surgery (83.2 \pm 15.9 vs. 87.1 $\pm 21.7 \mathrm{~min}$, in $E$ and L, respectively), intraoperative blood loss (15.2 $\pm 7.5 \mathrm{vs}$. $17.3 \pm 11.1 \mathrm{ml}$, respectively), median hospital stay, or the time to pass flatus and stool. The difference in the time needed to start the adjuvant treatment (38.7 \pm 5.7 vs. $33.2 \pm 5.8$ days, $p<0.01$ ), was compensated by the reduction of time living with a stoma (17.2 vs. 299.0 days) and health care costs: (43.68 vs. 698.42 USD).

Conclusions: Early closure is a safe and effective therapeutic approach, improving the recovery. Therefore it should be implemented as part of the ERAS protocol for rectal cancer patients.
\end{abstract}

Key words: protective stomy, colorectal cancer, loop ileostomy.

\section{Introduction}

Colorectal cancer is the second most common cause of death due to malignant disease in Europe, and surgery remains a key part of its treatment [1]. The Enhanced Recovery After Surgery (ERAS) proto- col was introduced into surgical practice more than a decade ago, and changed the clinical outcomes almost immediately [2-4].

Nonetheless, there is still room for improvement, particularly in case of rectal cancer. Its treatment depends on the location and stage; however, all pa-

\section{Address for correspondence}

Prof. Stanisław Kłęk, General and Oncology Surgery Unit, Stanley Dudrick’s Memorial Hospital, 15 Tyniecka St, 32-050 Skawina, Poland, fax: +48 604293 566, e-mail: klek@poczta.onet.pl 
tients with resectable tumors would undergo surgery either after neoadjuvant radio- and chemotherapy or as the first step of the treatment. One of the most common and most dangerous complications is the anastomotic leak $(\mathrm{AL})$ [5-17]. There are several effective methods to reduce its risk, the majority of them being covered by parts of the ERAS protocol, but there only a few which may improve the anastomotic healing from a more technical than general point of view. One of the key techniques is the protective (or 'defunctioning') loop ileostomy (PLI). It was proven to be an effective, yet obviously burdensome procedure $[18,19]$. The main drawbacks of PLI include reduction of the patient's quality of life and increase of chemotherapy's complication rate, often leading to dose reduction or regimen modification [20]. Most centers restore the continuity of the gastrointestinal tract more than one month after the adjuvant chemotherapy is terminated [21]. That approach is burdensome not only for patients, whose quality of life is significantly lower, but also for the health care system, which must cover the direct costs of stoma bags and indirect expenses of social inactivity. It also contradicts to some extent the ERAS concept of perfecting the post-surgical recovery period.

So-called 'early closure' (EA) can provide a solution to the problem. It can be safely performed within days or weeks after the primary surgery and it is an effective procedure [22-25]. Undoubtedly, it could be a valuable asset to ERAS, yet it has not been recognized that way.

\section{Aim}

The aim of the randomized, controlled clinical trial was to prove that EA should become a part of the routine ERAS approach, as it improves the quality of life and speeds up recovery, leading either to the termination of successful surgical treatment or enabling the start of adjuvant treatment.

\section{Material and methods}

A randomized, controlled clinical trial was performed at two surgical units between October the $1^{\text {st }}, 2016$ and December the $31^{\text {st }}, 2017$. Both centers have been using the ERAS protocol in daily routine practice for more than 5 years. The compliance rate with ERAS guidelines for both of them reaches $>90 \%$ every year.

Fifty-eight adult rectal cancer patients (24 females, 34 males, mean age 55.7 and 56.2) with PLI created during the low anterior section were randomly assigned to two study groups: early closure ( $E, n=29$ ), late closure (L, $n=29$, control group).

Randomization was performed before discharge from the hospital by a person not involved in the study using sealed envelopes containing computer-generated allocation numbers. No blocking or stratification was used.

Inclusion criteria: $\geq 18$ years of age, rectal adenocarcinoma, anterior resection of the rectum with PLI. Exclusion criteria: colorectal malignancy other than rectal adenocarcinoma, PLI performed for other indications (for example as part of treatment for postoperative complication), lack of informed consent.

Patients were well matched with regard to age, gender, nutritional status (BMI), and diagnosis. Detailed characteristics are presented in Table I.

All patients were treated with neoadjuvant radiochemotherapy (short and long arm) before surgery. Surgical complications were described and then presented using the Clavien-Dindo classification [26].

As the improvement of the quality of life achieved by reconstruction of the gastrointestinal tract was obvious and does not require any additional studies, the working hypothesis assumed that EC is safe and does not increase the complication rate (primary end point). The secondary objective (secondary end point) was to assess the impact of EC on the start of postoperative adjuvant anti-cancer treatment.

It was assumed that the confirmation of the complete has to be healing not only clinical, but also pathological. The evaluation of all anastomoses was performed by a skilled pathologist.

The surgical technique was the same for both groups and was composed of the following phases: - excision of the existing stoma,

- excision of the last $1 \mathrm{~cm}$ of the small intestine on both sides of resection,

- end-to-end anastomosis with double layer continuous running suture (Vicryl 4-0),

- fascia closure with continuous running suture (PDS II, 0),

- skin wound closure.

The postoperative care after both procedures was based on the enhanced recovery after surgery (ERAS) protocol. Major surgical aspects of ERAS included: no drainage, no nasogastric tube, no osmotic bowel preparation, single shot antibiotics $30 \mathrm{~min}$ before skin incision, mobilization directly after surgery, less than $500 \mathrm{ml}$ of $i . v$. intravenous fluids on the 
Table I. General characteristics and nutritional parameters

\begin{tabular}{|c|c|c|c|}
\hline Parameter & Early closure (E) & Late closure (L) & $P$-value \\
\hline Number of patients & 29 & 29 & - \\
\hline Females, $n(\%)$ & $11(37.9)$ & $13(44.8)$ & 0.5941 \\
\hline Males, $n(\%)$ & $18(62.1)$ & $16(55.2)$ & \\
\hline Age, mean \pm SD (median) [years] & $55.7 \pm 12.2(54)$ & $56.2 \pm 12.5(58)$ & 0.6217 \\
\hline \multicolumn{4}{|l|}{ Stage of cancer, $n(\%)$ : } \\
\hline$\| A$ & $8(27.6)$ & $9(31.0)$ & 0.9301 \\
\hline $\mathrm{IIB}$ & $2(6.9)$ & $3(10.3)$ & \\
\hline IIC & $7(24.1)$ & $5(17.3)$ & \\
\hline IIIA & $4(13.8)$ & $3(10.3)$ & \\
\hline$\| I I B$ & $5(17.3)$ & $4(13.8)$ & \\
\hline IIIC & $3(10.3)$ & $5(17.3)$ & \\
\hline Median length of hospital stay after primary resection (IQR) [days] & $6(6-7)$ & $6(5-7)$ & 0.4455 \\
\hline Type of primary surgery, $n(\%):$ & & & - \\
\hline Laparoscopic & $26(89.7)$ & $26(89.7)$ & \\
\hline Open (after conversion) & $3(10.3)$ & $3(10.3)$ & \\
\hline Neoadjuvant radio-chemotherapy, $n(\%)$ & $29(100)$ & $29(100)$ & - \\
\hline Adjuvant chemotherapy, $n(\%)$ & $25(86.2)$ & $27(93.1)$ & 0.3884 \\
\hline
\end{tabular}

* $\chi^{2}$ test with Yates' correction.

$1^{\text {st }}$ day postoperatively, no opioid-based pain killers. The primary rectal resection was performed laparoscopically or with open technique after conversion due to technical difficulties. All ileo-rectal anastomoses were created using a stapling device (Premium Plus CEEA 29, Johnson \& Johnson, USA).

Nutritional aspects: beginning of oral food intake - 2-4 h after surgery, regular food on postoperative day 1 . All patients were discharged from hospital between the $3^{\text {rd }}$ and $5^{\text {th }}$ day.

Timing of the reconstruction:

1) Early closure group: the patient was re-admitted to the hospital 14 days after the discharge after the primary resection. The primary discharge took place between the $4^{\text {th }}$ and $6^{\text {th }}$ postoperative day.

2) Late closure group: the patient was re-admitted to the hospital 30 days after the termination of the adjuvant chemotherapy, usually approximately 7 months after the primary resection. The primary discharge was the same as for the EC group.
In all patients the sigmo-rectal anastomosis was checked with endoscopy. The distal loop of the protective stoma was flushed with $1000 \mathrm{ml}$ of saline the day before the restoration to prove the effective passage of the gastrointestinal tract.

\section{Ethics and consent}

The Ethics Committee of the Jagiellonian University Medical School approved the study [122.6120.207.2016]. Patients were personally enrolled and approached by the lead investigators (SK, JS). Informed written consent was obtained from each participant before enrollment. The study was carried out following the international ethical recommendations stated in the Helsinki Declaration and was registered under the number: ClinicalTrials. gov PRS registration: NCT02997267.

\section{Statistical analysis}

All data were analyzed with StatSoft Statistica v.12. The results are presented as mean \pm stan- 


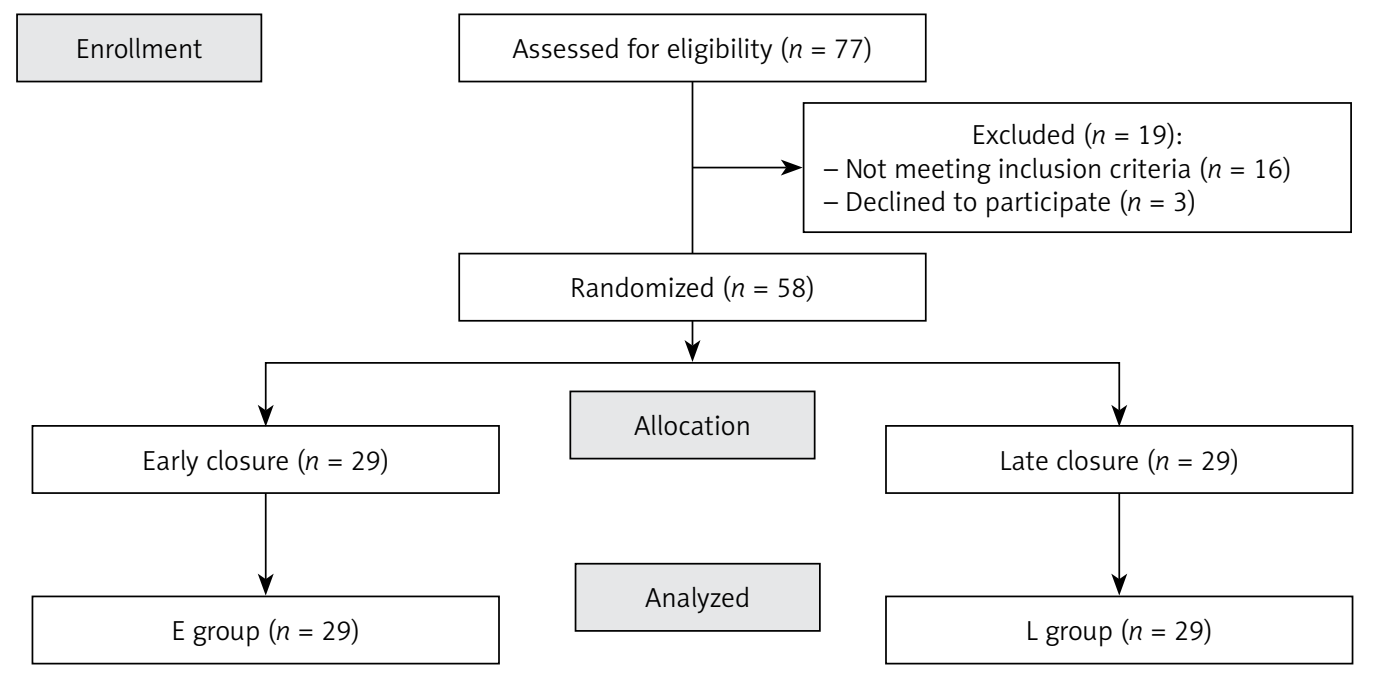

Figure 1. CONSORT diagram

dard deviation (SD), median and interquartile range (IQR) when appropriate. The study of categorical variables used the $\chi^{2}$ test of independence. The Shapiro-Wilk test was used to check for normal distribution of data. Student's t-test was used for normally distributed quantitative data. For non-normally distributed quantitative variables, the Kruskal-Wallis test was used. In the next step the Friedman test was used for non-normally distributed dependent variables. Results were considered statistically significant when the $p$-value was found to be less than 0.05 .

To calculate the sample size we decided to use the time to start the adjuvant chemotherapy as the main parameter. We assumed that a delay of three or more weeks at the start of chemotherapy would represent a clinically relevant difference. To detect that we calculated that 23 patients would be required in each treatment arm to give the study the power of $90 \%$.

Table II. Postoperative complications

\begin{tabular}{|lcc|}
\hline $\begin{array}{l}\text { Grade of } \\
\text { complication acc. } \\
\text { to Clavien-Dindo }\end{array}$ & $\begin{array}{c}\text { Early closure } \\
\text { group (E) }\end{array}$ & $\begin{array}{c}\text { Late closure } \\
\text { group (L) }\end{array}$ \\
\hline I & 2 (SSI) & 3 (SSI) \\
\hline II & 0 & 0 \\
\hline IIIa & 0 & 0 \\
\hline IV & $\begin{array}{c}\text { 1 (strangulation } \\
\text { and ileus) }\end{array}$ & $\begin{array}{c}\text { 1 (intraabdominal } \\
\text { bleeding) }\end{array}$ \\
\hline
\end{tabular}

\section{Results}

Fifty-eight patients were fully evaluated; there were no drop-outs. The CONSORT diagram shows patients' flow through the study (Figure 1). Neither fatal outcomes nor anastomotic leaks were observed in either group. Postoperative complications included volvulus (group $\mathrm{E}, n=1$ ), surgical site infection ( $\mathrm{E}=2, \mathrm{~T}=3, p>0.05)$ and intraabdominal bleeding (group $\mathrm{T}, n=1$ ) (Table II).

Time to pass flatus and the first stool was almost the same in both groups (2 days for both groups). There were no significant differences between the two groups regarding either the length of surgery ( $T=83.2$ in $\mathrm{E}$ vs. $87.1 \mathrm{~min}$ in $\mathrm{L}, p>0.05$ ) or intraoperative blood loss ( $E=15.2 \mathrm{ml}$ vs. $\mathrm{L}=17.3 \mathrm{ml}$, $p>0.05)$. The median hospital stay was 5 days in both groups $(p>0.05)$.

The mean time to start adjuvant treatment was $38.7 \pm 5.7$ and $33.2 \pm 5.8$ days in groups $E$ and $L$, respectively, which was statistically significant, but without any clinical importance, as the adjuvant treatment started within 8 weeks after resection. The mean duration of living with a stoma was 17.3 days in group $E$ and 278.6 days in group L. The mean health care costs (calculated as the cost of stoma bags) per capita per period of treatment were: PLN 152.9. in group E and PLN 2413.1 group L (USD 43.68 vs. 698.42). A detailed description is presented in Table III.

\section{Pathologist's report}

Microscopic examination revealed no significant differences between the two groups of patients. In 
Table III. Clinical outcomes

\begin{tabular}{|lccc|}
\hline Parameter & $\begin{array}{c}\text { Early closure } \\
\text { group (E) }\end{array}$ & $\begin{array}{c}\text { Late closure } \\
\text { group (L) }\end{array}$ & $P$-value \\
\hline Patients with complications, $n$ (\%) & $3(10.3)$ & $4(13.8)$ & 0.6873 \\
\hline Time to first flatus, median (IQR) [days] & $2(1-2)$ & $2(1-2)$ & 0.9431 \\
\hline Time to first defecation, median (IQR) [days] & $3(2-3)$ & $3(2-3)$ & 0.5934 \\
\hline Length of hospital stay, median (IQR) [days] & $5(4-6)$ & $5(4-5)$ & 0.6316 \\
\hline Readmission, $n$ (\%) & 0 & 0 & - \\
\hline Duration of surgery, mean \pm SD [min] & $83.2 \pm 15.9$ & $87.1 \pm 21.7$ & 0.4967 \\
\hline Perioperative blood loss, mean \pm SD [ml] & $15.2 \pm 7.5$ & $17.3 \pm 11.1$ & $<.7142$ \\
\hline Number of stoma bags/treatment period, mean \pm SD (median) & $19.1 \pm 2.2(17)$ & $307.6 \pm 91.9(305)$ & $<.001$ \\
\hline Duration of living with stoma, mean \pm SD (median) [days] & $17.3 \pm 1.5(17)$ & $278.6 \pm 89.1(250)$ & $<.001$ \\
\hline Cost of stoma bags/treatment period, mean \pm SD (median) & $152.9 \pm 16.3(148)$ & $2413.1 \pm 759(2320)$ & $<0.001$ \\
\hline Time to start adjuvant chemotherapy, mean \pm SD (median) [days] & $38.7 \pm 5.7(41)$ & $33.2 \pm 5.8(31)$ & $<0.001$ \\
\hline
\end{tabular}

reviewed slides from group E patients' signs of acute tissue injury were present. Areas of granulation tissue, focal hemorrhages and inflammatory infiltrates containing numerous neutrophils located especially in the intestinal mucus membrane were observed. Tissue samples obtained from group $L$ exhibited acute-on-chronic pattern of inflammation. Mononuclear inflammatory infiltrates with various admixtures of neutrophils were present in lamina propria of the intestinal mucus membrane. In 1 case neutrophils entered crypt epithelium and formed clusters in the glandular lumen. Additionally, superficial erosions, hyperemia and fibrosis of the submucosal membrane were observed. In both groups intestinal epithelium and adjacent epidermis focally showed slight reactive changes.

\section{Discussion}

The ERAS protocol has been the major gamechanger as far as perioperative care is considered. Being a protocol composed of approximately 17 key aspects, each improving the clinical outcome, it influences all facets of surgery. The ERAS has still been developing and improving over the years, reaching for new areas of improvement, such as the minimally invasive approach, new combinations of drugs and interventions. Practically each new clinical approach can be considered as part of the ERAS protocol, as long as it speeds up the recovery.
Anastomotic leakage (AL) is a major problem in colorectal surgery, particularly in rectal cancers located not far from the anal verge [5-9]. It has been observed with the frequency ranging from $2.6 \%$ to $19.0 \%$ [5-15]. AL is the a main reason for the increase in the postoperative morbidity, mortality and worsening of the quality of life, and it may be a reason for an increase in the local recurrence of rectal cancer [5-17].

The protective loop ileostomy was introduced to divert the fecal stream from a more distal anastomosis in order to reduce the consequences of any anastomotic leakage $[18,19]$. The intervention proved to be effective $[18,19]$.

From patients' perspective, however, the quality of life when living with a stoma cannot be satisfactory. Therefore the need for gastrointestinal (GI) tract reconstruction is unquestionable [20]. The question when to do it, however, remains open. As the surgery during chemotherapy is burdened with a higher rate of complications, almost all surgeons would wait until the adjuvant treatment is terminated. Reasonable as it is, that approach exposes the patient to several months of living with a stoma, leading to progressive health care costs due to equipment, additional medical care and social inactivity.

For these reasons, it would seem judicious to close the stoma as early as possible [21-23]. The safety and efficacy of the early procedure was confirmed in the four-study metaanalysis by Robertson et al. [20]. 
EC poses two clinical risks: unsuccessful anastomotic healing and the delay of adjuvant chemotherapy. EC allows also to elevate the rectal cancer patient to a person of maximally accelerated recovery.

For many years, however, there was an open issue of the optimal timing; some authors, such as Menegaux et al. and Danielsen et al., proposed to close it between the $8^{\text {th }}$ and the $13^{\text {th }}$ postoperative day [22, 23]. None of them, however, presented either the precise timing or the rationale for that time period [24].

The first question was solved by the study of Marjnovic and Hopt [25]. These authors demonstrated that the healing process is composed of three phases: inflammatory, proliferative and reparatory [25]. The first phase (postoperative days 1 to 4; POD $1-4$ ) is dominated by inflammation, migration of immune cells and the activity of mucosa, and for these reasons it is mostly surgical technique dependent. The second (POD 5-14) is mostly based on collagen formation and angiogenesis, and decides about the quality of the juncture [25]. During the third one (up to 12 months after surgery), the re-modeling of the anastomosis takes place.

From the clinical point of view, the first two phases are the most important, and when they are finished, the anastomosis is safe. In our study there was a small difference in the time needed to start the adjuvant treatment (38.7 vs. 33.2 days, $p<0.01$ ). Nonetheless, as the adjuvant chemotherapy should start not later than 8 weeks after surgery, because the later one may lead to a decrease of 5-year survival rates [26-29], the difference observed in our study was insignificant from the clinical perspective. Therefore, if EC is supposed to be a reliable clinical option, it can be performed within a few weeks after the primary resection. Thanks to studies on Enhanced Recovery After Surgery (ERAS) protocols, the primary discharge can take place on POD 4 to 6 [3032], enabling fortnight readmission for EC.

The current study proved that EC can be safe (no differences in the rate of complications, length of stay) and effective (almost the same time to pass flatus and stool, no fistulization).

Pathological examination did not reveal major differences between groups, which confirmed that the healing process was already accomplished. It was reflected in technical considerations: the duration of surgery and intraoperative blood loss were the same.
The 14-day post-discharge GI restoration led to significant health care savings - mean health care costs per capita per period of treatment were: PLN 136.1 in group E and PLN 2440.0 in group L (34 vs. 610 USD). Moreover, the reduction of stoma days resulted in higher quality of life and facilitated the uncomplicated course of adjuvant chemotherapy, which started not later than 6 weeks after primary surgery.

\section{Conclusions}

Early closure of protective ileostomy is a safe and effective therapeutic approach. It allows one to reduce health care costs and improve the quality of life, not exposing the further treatment to a risk of failure. For the above reasons it should be implemented as part of the ERAS protocol for rectal cancer patients.

\section{Conflict of interest}

The authors declare no conflict of interest.

\section{References}

1. Sant M, Allemani C, Santaquilani M, et al. Survival of cancer patients diagnosed in 1995-1999. Results and commentary. Eur J Cancer 2009; 45: 931-91.

2. Gustafsson UO, Oppelstrup H, Thorell A, et al. Adherence to the ERAS protocol is associated with 5-year survival after colorectal cancer surgery: a retrospective cohort study. World I Surg 2016; 40: 1741-7.

3. Fearon KC, Ljungqvist O, Von Meyenfeldt M, et al. Enhanced recovery after surgery: a consensus review of clinical care for patients undergoing colonic resection. Clin Nutr 2005; 24: 466-77.

4. Gustafsson UO, Scott MJ, Schwenk W, et al. Guidelines for perioperative care in elective colonic surgery: Enhanced Recovery After Surgery $\left(\right.$ ERAS $\left.^{\circledR}\right)$ Society recommendations. Clin Nutr 2012; 31: 783-800.

5. Jannasch O, Klinge T, Otto R, et al. Risk factors, short and long term outcome of anastomotic leaks in rectal cancer. Oncotarget 2015; 34: 36884-93.

6. Klein M. Postoperative non-steroidal anti-inflammatory drugs and colorectal anastomotic leakage. NSAIDs and anastomotic leakage. Dan Med J 2012; 59: B4420.

7. Brown SR, Mathew R, Keding A, et al. The impact of postoperative complications on long-term quality of life after curative colorectal cancer surgery. Ann Surg 2014; 259: 916-23.

8. Bertelsen CA, Andreasen AH, Jorgensen T, et al. Anastomotic leakage after curative anterior resection for rectal cancer: short and long term outcome. Colorectal Dis 2010; 12: 76-81.

9. Bertelsen CA, Andreasen AH, Jorgensen T, et al. Anastomotic leakage after anterior resection for rectal cancer: risk factors. Colorectal Dis 2010; 12: 37-43. 
10. den Dulk M, Marijnen CA, Collette L, et al. Multicentre analysis of oncological and survival outcomes following anastomotic leakage after rectal cancer surgery. Br J Surg 2009; 96: 1066-75.

11. Penninckx F. Anastomotic leakage: a disaster or a challenge with an impact on survival after rectal cancer surgery? Colorectal Dis 2011; 13: 237-8.

12. Richards $\mathrm{CH}$, Campbell V, Ho C, et al. Smoking is a major risk factor for anastomotic leak in patients undergoing low anterior resection. Colorectal Dis 2012; 14: 628-33.

13. Sajid MS, Ahamd A, Miles WF, B et al. Systematic review of oncological outcomes following laparoscopic vs open total mesorectal excision. World J Gastrointest Endosc 2014; 6: 209-19.

14. Damen N, Spilsbury K, Levitt M, et al. Anastomotic leaks in colorectal surgery. ANZ J Surg 2014; 84: 763-8.

15. Eriksen MT, Wibe A, Norstein J, et al. Anastomotic leakage following routine mesorectal excision for rectal cancer in a national cohort of patients. Colorectal Dis 2005; 7: 51-7.

16. McDermott FD, Heeney A, Kelly ME, et al. Systematic review of preoperative, intraoperative and postoperative risk factors for colorectal anastomotic leaks. Br J Surg 2015; 102: 462-79.

17. Vermeer TA, Orsini RG, Daams F, et al. Anastomotic leakage and presacral abscess formation after locally advanced rectal cancer surgery: incidence, risk factors and treatment. Eur J Surg Oncol 2014; 40: 1502-9.

18. Chude GG, Rayate NV, Patris V, et al. Defunctioning loop ileostomy with low anterior resection for distal rectal cancer: should we make an ileostomy as a routine procedure? A prospective randomized study. Hepatogastroenterology 2008; 55: 1562-7.

19. Huser N, Michalskie C, Erkan M, et al. Systematic review and metaanalysis of the role of the defunctioning stoma in low rectal cancer. Ann Surg 2008; 248: 52-60.

20. Robertson JP, Wells $\mathrm{Cl}$, Vather R, et al. Effect of diversion ileostomy on the occurrence and consequences of chemotherapy-induced diarrhea. Dis Colon Rectum 2016; 59: 194-200.

21. Thalheimer A, Bueter M, Kortuem M, et al. Morbidity of temporary loop ileostomy in patients with colorectal cancer. Dis Colon Rectum 2006; 49: 1011-7.

22. Menegaux F, Jordi-Galais P, Turrin N, et al. Closure of small bowel stomas on postoperative day 10. Eur J Surg 2002; 168: 713-5.

23. Danielsen AK, Park J, Jansen JE, et al. Early closure of a temporary ileostomy in patients with rectal cancer: a multicenter randomized controlled trial. Ann Surg 2017; 265: 284-90.

24. Robertson JP, Puckett J, Vather R, et al. Early closure of temporary loop ileostomies: a systematic review. Ostomy Wound Manage 2015; 61: 50-7.

25. Marjanovic G, Hopt TH. Physiologie der Anastomosenheilung. Der Chirurg 2011; 82: 41-7.

26. Des Guetz G, Nicolas P, Perret GY, et al. Does delaying adjuvant chemotherapy after curative surgery for colorectal cancer impair survival? A meta-analysis. Eur J Cancer 2010; 46: 1049-55.

27. Biagi JJ, Raphael MJ, Mackillop WJ, et al. Association between time to initiation of adjuvant chemotherapy and survival in colorectal cancer: a systematic review and meta-analysis. JAMA 2011; 305: 2335-42.

28. Day AR, Middleton G, Smith RV, et al. Time to adjuvant chemotherapy following colorectal cancer resection is associated with an improved survival. Colorectal Dis 2014; 16: 368-72.
29. Clavien PA, Barkun J, de Oliveira ML, et al. The Clavien-Dindo classification of surgical complications: five-year experience. Ann Surg 2009; 250: 187-96.

30. Gustafsson UO, Scott MJ, Schwenk W, et al. Guidelines for perioperative care in elective colonic surgery: Enhanced Recovery After Surgery (ERAS) Society recommendations. World J Surg 2013; 37: 259-84.

31. Nygren J, Thacker J, Carli F, et al. Guidelines for perioperative care in elective rectal/pelvic surgery: Enhanced Recovery After Surgery (ERAS) Society recommendations. World I Surg 2013; 37: 285-305.

32. Pędziwiatr M, Pisarska M, Kisielewski M, et al. ERAS protocol in laparoscopic surgery for colonic versus rectal carcinoma: are there differences in short-term outcomes? Med Oncol 2016; 33: 56-9.

Received: 30.09.2018, accepted: 15.10.2018. 